\title{
Diagnostic and Treatment Modalities for All Localizations of Ectopic Pregnancy
}

\author{
Ibrahim Alkatout ${ }^{{ }^{*}}$, Ulrich Honemeyer ${ }^{2}$, Karl-Günter Noé ${ }^{3}$, Christel Eckmann-Scholz ${ }^{1}$, Nicolai Maass ${ }^{1}$, \\ Mohamed Elessawy ${ }^{1}$, Liselotte Mettler ${ }^{1}$
}

\begin{abstract}
Objectives: The rate of ectopic pregnancies has increased from $0.5 \%$ in 1970 to $2 \%$ today. With the growing application of imaging techniques, however, all normal and abnormal implantations can now be detected early. This review article tries to assess a workup of all localizations of human ectopic pregnancies.

Materials and Methods: All diagnostic and therapeutic modalities from the non-medical conservative method, to the medical nonsurgical options through to the surgical laparoscopic approach for the treatment of ectopic pregnancies are assessed in this review. Results: Observational treatment: Monitoring HCG levels until tubal abortion or resorption is a treatment option with the risk of failure and requires patience from the patient and the treating physician. Medical treatment: The predominant drug is methotrexate but other systemic drugs, such as actinomycin D, prostaglandins and RU 486, can also be applied. Surgical treatment: In the case of tubal pregnancies, salpingotomy, partial salpingectomy followed by laparoscopic anastomosis or fimbrial milking is performed to preserve tubal function. According to their localization non-tubal ectopic pregnancies (ovarian pregnancy, ectopic abdominal pregnancy, interstitial or cornual pregnancy/rudimentary horn, intraligamental and cervical pregnancies) all require their own specific treatment.

Conclusion: Today, ectopic pregnancies are diagnosed early enough to be treated effectively by laparoscopic surgery. In 5\%-15\% of women the remaining positive HCG values in serum after treatment refer to remnant conception products and may be treated with a final methotrexate injection or expectantly.

Keywords: Ectopic pregnancy, Laparoscopy, Methotrexate, Tubal function, Non-tubal ectopic pregnancy, Early diagnosis, Individualized treatment
\end{abstract}

\section{Introduction}

After fertilization in the ampullary part of the fallopian tubes, the growing morula moves in a circular fashion for 4-5 days through the tube towards the uterine cavity while differentiating into the embryoblast and trophoblast (1). Today, $2 \%$ of early pregnancies implant in extrauterine sites. They can be removed safely by laparoscopic surgery. The histological examination (Figure 1) is always required. The prevalence of ectopic pregnancy in all women presenting with first-trimester bleeding, lower abdominal pain or a combination of the two to an emergency department is between $6 \%$ and $16 \%$. (2)

The tubal diameter increases from the tubouterine junction to the fimbria, but remains a narrow pathway. This may be the reason why $98 \%$ of all ectopic pregnancies are located in the fallopian tube (ampulla 64\%, isthmus $25 \%$, infundibulum $9 \%$ and interstitial $2 \%$ ), $0.5 \%$ in the ovary and $0.1 \%$ intraabdominal. Furthermore, the ampullary region is the most distal place where ascending infections can cause phymosis and occlusion. Less than
$2 \%$ of ectopic pregnancies are located intrauterine $(0.4 \%$ cervical and $<1 \%$ corneal).

Today, the mortality rate of ectopic pregnancies worldwide is estimated to be 3.8 per 1000 ectopics. Early diagnosis before the occurrence of hemoperitoneum and/or hypovolemic shock leads to a striking decrease of mortality and to a better recognition at the initial medical assessment (2). Owing to the advances made in transvaginal ultrasound, radio immunoassays for serum $ß-H C G$ levels and an increased vigilance by clinicians with more experience of diagnostic laparoscopy, more than $80 \%$ of ectopic pregnancies are now diagnosed intact and offer an opportunity for conservative non-medical or medical non-surgical treatment (3-5).

\section{Material and Methods}

The Implantation Window

Any impedance of the conceptus migration to the uterine cavity may precipitate an ectopic gestation. The major contributing risk factors for ectopic pregnancy 


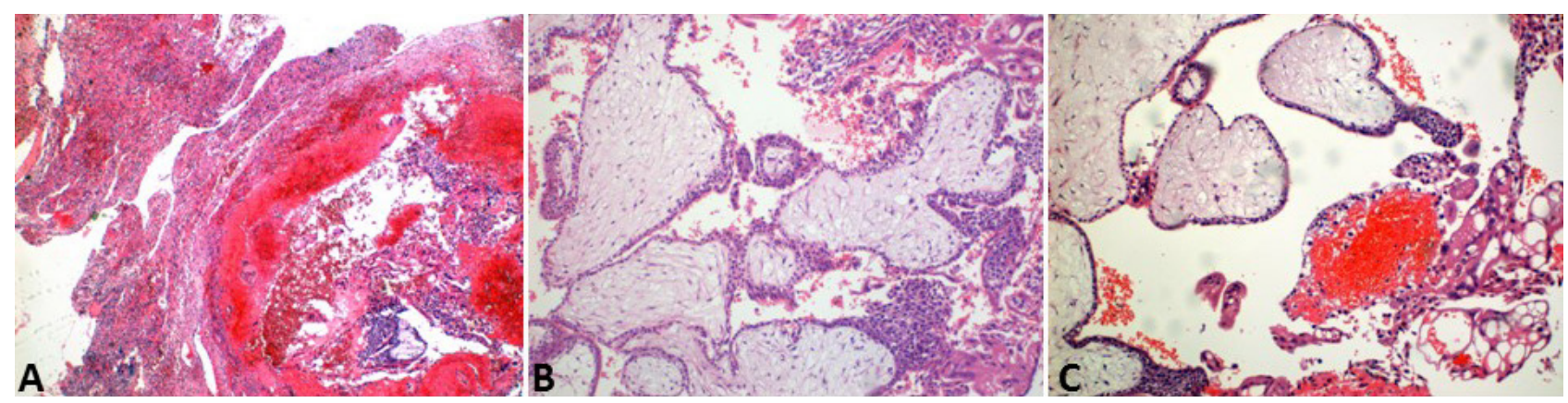

Figure 1. (A) Gestational material next to hemorrhage and fibrin exudation as well as regressive altered villi. (B) Trophoblastic giant cells next to regressive altered villous stroma, trophoblast, and decidual stroma. (C) Regressive altered villous stroma, trophoblast, hemorrhage and fibrine, trophoblastic giant cells.

are the following: previous tubal surgery, history of ectopic pregnancy, sexually transmitted disease, pelvic inflammatory disease, in utero diethylstilboestrol exposure, history of infertility, anatomical uterine/ tubal abnormality, previous tubal ligation, previous or current IUD use, assisted reproductive technologies, current smoking, non-white (all races other than white), age between 35 and 44 (compared to those from 15 to 24 ), induced abortion, T-shaped uterus, myomata and progestin-only contraceptives. Previous surgeries or infections, anatomic defects in the tubal epithelium, hormonal factors that interfere with normal transport of the conceptus and pathologic conditions that affect normal tubal function may arise. Estrogens and progestogens show different hormonal effects on the growth and movement of the epithelial tubal ciliae. The influence of smoking also has to be considered $(6,7)$.

Diagnostics - Pre-treatment Assessment of Ectopic Pregnancies

Additional unspecific parameters after a positive B-HCG are a slightly enlarged uterus, vaginal bleeding or spotting, pelvic pain and a palpable adnexal mass. Apart from mimicking the symptoms of other gynecological and even non-gynecological diseases, ectopic pregnancies appear in many variations often causing no pain at vaginal examination. Severe pain can also be experienced in a normal pregnancy.

Breakthrough bleedings appear as spotting because these ectopic pregnancies show only small HCG levels which do not support the continuous decidual transformation of the endometrium. The earliest appearance of symptoms occurs in the sixth week after the last period.

\section{Serum Level of $\beta-H C G$}

HCG starts with secretion on day 5 to day 8 . A serum array detects levels as low as $5 \mathrm{mIU} / \mathrm{mL}$ while the detection limit in urine is $20-50 \mathrm{mIU} / \mathrm{mL}$. The $\beta-H C G$ levels double every 1.5 days in the first 5 weeks of a regular gestation and every 3.5 days after 7 weeks.

Normal HCG levels for the time of pregnancy are only found in $30 \%$ of ectopics and the daily doubling increase of $B-H C G$ is lacking. An abnormal $B-H C G$ pattern is highly suspicious for an ectopic gestation or a no longer intact gestation (8-10).

\section{Imaging Signs in Ultrasound Scan}

At a gestational age of about 5 weeks, a normally developing gestational sac can be visualized showing an ovoid collection of fluid clinging to the endometrium as well as a yolk sac of $8 \mathrm{~mm}$ or more in diameter. However, a pseudogestational sac can mimic a gestational sac at an early stage. The pseudo gestational sac is often situated in the centre of the uterine cavity. Its margin is homogenous and round but it is difficult to define its boundary to the outside tissue. By contrast, a physiologic gestational sac is asymmetric and contains two concentric rings, separated by a thin echogenic layer. At a gestational age of 6-6.5 weeks the embryonic structures measure $4-5 \mathrm{~mm}$ and cardiac activity might already be detected.

At a serum B-HCG level of $1500 \mathrm{mIU} / \mathrm{mL}$, an intrauterine chorionic sac can be detected. The "Comet sign" of intervillous flow in power Doppler (PD) assessment of the decidua around the double echogenic ring and visualization of a yolk sac at 5 weeks plus, confirm the impression of an intrauterine implantation. Color-coded flow signals of the ectopic pregnancy are clearly separated from ovarian tissue and corpus luteum. The extent of vascularity reflects trophoblastic vitality and invasiveness (neo-angiogenesis), enhanced by vasodilatation of the fallopian vessels under the influence of maternal progesterone (11).

In $85 \%$ of all ectopic pregnancies the corpus luteum is found ipsilateral. Luteal color or PD flow may be used as a guide while searching for an ectopic pregnancy, and could be called the "lighthouse effect" of corpus luteum which directs the investigator to the color Doppler signals of the ectopic pregnancy (Table 1) (12-16).

The combination of transvaginal ultrasonography and serial quantitative B-HCG serum levels detects with a sensitivity of $96 \%$ and a specificity of $97 \%$ an ectopic pregnancy and remains the gold standard as well as the most cost-effective strategy for diagnosing ectopic pregnancy. A positive fetal heart beat is often absent because extrauterine pregnancies seldom develop vitally and only display an agglomeration of trophoblast tissue 
Table 1. Ultrasound Imaging Findings in Early Ectopic Pregnancy

\begin{tabular}{|c|c|c|}
\hline Ultrasound imaging findings & Sensitivity & Specificity \\
\hline Abdominal ultrasound (13) & $81 \%$ & $77 \%$ \\
\hline \multicolumn{3}{|c|}{ Transvaginal Ultrasound } \\
\hline No intrauterine gestational sac (14) & $100 \%$ & $89 \%$ \\
\hline Non-cystical adnexal mass (15) & $84-90 \%$ & $94 \%-95 \%$ \\
\hline Separate from ovary & $93 \%$ & $99 \%$ \\
\hline Cardiac activity & $20 \%$ & $100 \%$ \\
\hline Yolk sac or embryo & $37 \%$ & $100 \%$ \\
\hline Tubal ring/Yolk sac or embryo & $65 \%$ & $99 \%$ \\
\hline Tubal pregnancies (15) & $99 \% \%$ & $87 \%$ \\
\hline \multicolumn{3}{|l|}{ Fluid in the pouch of Douglas (15) } \\
\hline Any & $63 \%$ & $69 \%$ \\
\hline Echogenic & $56 \%$ & $96 \%$ \\
\hline Color-flow Doppler (15) & $95 \%$ & $98 \%$ \\
\hline \multicolumn{3}{|c|}{ Specific Findings (Appearance in \% of Cases) } \\
\hline Blob sign (inhomogeneous mass) (16) & & $60 \%$ \\
\hline Bagel sign (hyperechoic ring) (16) & & $20 \%$ \\
\hline Obvious gestational sac fetal pole with/without fetal cardiac activity (16) & & $13 \%$ \\
\hline Single test diagnosis vs. entire findings of ultrasound & & $74 \%$ \\
\hline
\end{tabular}

and a surrounding hematoma. (16-18).

\section{Progesterone Levels}

The progesterone serum level has a sensitivity of $15 \%$ to detect pregnancy failure. As a stable marker in the first trimester, a serum level above $22 \mathrm{ng} / \mathrm{mL}$ speaks for a viable intrauterine pregnancy whereas levels under $5 \mathrm{ng} / \mathrm{mL}$ are indicative of a nonviable pregnancy $(18,19)$.

Uterine diagnostic curettage and the puncture of the pouch of Douglas have become obsolete as diagnostic procedures (18).

This straightforward diagnosis of ectopic pregnancy is based on three pillars. Symptoms that can be determined during physical examination, clinical features that appear in ultrasound scan and laboratory tests that can substantiate a primary suspicion (Figure 2).

\section{Differential Diagnosis}

Ectopic pregnancy can be misdiagnosed by the following gynaecological differential diagnoses: early stage of normal pregnancy, miscarriage, intrauterine abortion, torsion of ovary or fallopian tube, ovarian cyst, ruptured corpus luteum cyst or follicle, necrotic myoma, pelvic inflammatory disease (salpingitis) and tubo-ovarian abscess. In addition, the following conditions, other than of gynaecological origin, can be misinterpreted as ectopic pregnancy: acute appendicitis, cystitis, pyelonephritis, nephrolithiasis, perforation of hollow organs e.g. stomach, bowel, gallbladder, obstruction of hollow organs, intraabdominal inflammation (peritoneum, all abdominal organs, diverticulum), rupture of parenchymatous organs (liver, spleen, kidney), vascular ischemic disease (bowel, mesenterium) and vascular hemorrhagic disease (aorta, all abdominal vessels) (20).

\section{Results}

Treatment of Ectopic Pregnancies

The treatment decision includes operative and medical management as well as the option not to treat and follow up with observation and clinical and laboratory tests. It is known that many early ectopic pregnancies result in spontaneous abortion and re-absorption and this might make aggressive treatment unnecessary. Medical treatment with methotrexate is restricted to a few limited indications (21).

\section{Expectant Treatment}

Ectopic pregnancies might have a natural tendency to

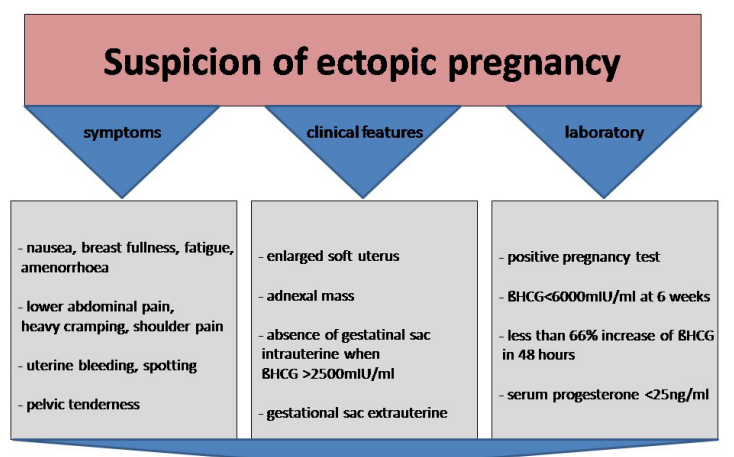

substantiation leads to laparoscopy

Figure 2. The 3 Pillars That Substantiate an Early Suspicion of Ectopic Pregnancy. 
terminate in tubal abortion or complete resorption. Modern monitoring might be sufficient and enhance the preservation of tubal function and fertility. We consider the following prognostic parameters: lack of clinical symptoms, a sonographic adnexal tumor below $4 \mathrm{~cm}$ with a decreasing tendency, less than $50 \mathrm{ml}$ of free fluid and an initial B-HCG level of below $2000 \mathrm{mU} /$ $\mathrm{mL}$ with a decreasing tendency after a 48-hour interval. However, more than $90 \%$ of all affected women with ectopic pregnancies develop increasing and endangering symptoms that lead to an operative intervention (4,22-25).

\section{Medical Treatment}

Today methotrexate as well as actinomycin D, potassium chloride, prostaglandins and RU 486 are applied. In view of the uncertainty of treatment success and possible adverse side effects, the indication for a conservative treatment has to be weighed up carefully.

Methotrexate can disrupt the rapidly-dividing trophoblastic cells. The expected time to resolution of the ectopic pregnancy is 3 to 7 weeks after methotrexate application. The selective use of methotrexate can be as effective as surgery although adverse side effects are possible, such as bone marrow suppression, elevated liver enzymes, rash, alopecia, stomatitis, nausea, diarrhea, and to a lower extent pleuritis, dermatitis, conjunctivitis, gastritis and enteritis. The success rate of methotrexate is up to $94 \%$. Nevertheless, this depends on the B-HCG level. The lower the serum level at the beginning of the therapy, the higher the success rate is.

The use of methotrexate to destroy an ectopic pregnancy has been advocated for women with an atypical localization in the cervix, interstitially, cornually or in the abdominal cavity, an incomplete resolution of a surgically treated ectopic gestation, residual trophoblast tissue or persisting low B-HCG levels after curettage with no evidence of trophoblast material in the histological examination. Furthermore, women with a high operative risk and contraindications to anesthesia, for example after induced ovarian hyperstimulation syndrome, and women who are expected to have extensive intraperitoneal abdominal adhesions might be optimal candidates for a preliminary medical treatment if they are hemodynamically stable (20).

Methotrexate can be given locally or systemically by intramuscular injection of $1 \mathrm{mg} / \mathrm{kg}$ or $50 \mathrm{mg} / \mathrm{m}^{2}$. Women with a hematocrit below $35 \%$ should take $325 \mathrm{mg}$ ferrous sulphate twice daily as they may bleed during the laparoscopic removal and afterwards. If the B-HCG levels do not decrease by at least $15 \%$ after 7 days, a second dose of methotrexate has to be given(20). For an overview on methotrexate treatment see Figure 3.

\section{Surgical Treatment}

In most cases of ectopic pregnancy surgical treatment is without doubt necessary(26).

Diagnostic laparoscopy - Evacuation of the hemoperitoneum - followed by Operative Laparoscopic Treatment in the Same Session

A diagnostic laparoscopy visualizes the location, size and nature of the ectopic pregnancy. A hemoperitoneum can complicate the whole operation. Depending on the degree of bleeding, a primarily inserted grasper localizes the ruptured tube and tamponades the bleeding site. After safe compression or preferably suture of the bleeding source, blood and blood clots can be removed by suction and irrigation $(27,28)$.

The following specific techniques can be applied dependent on the individual case.

\section{Surgical Treatment of Tubal Pregnancy} Milking of Tubal Pregnancy Through the Fimbrial End If the product of conception is located at the outer region of the fallopian tube or the fimbrial end, it can be removed by grasping the tubal segment and stepwise milking the gestational product out of the fimbriae of the tube. The product of conception is gently pushed until it is extruded. The stepwise movements begin in the proximal part and gently push the product into the abdominal cavity.

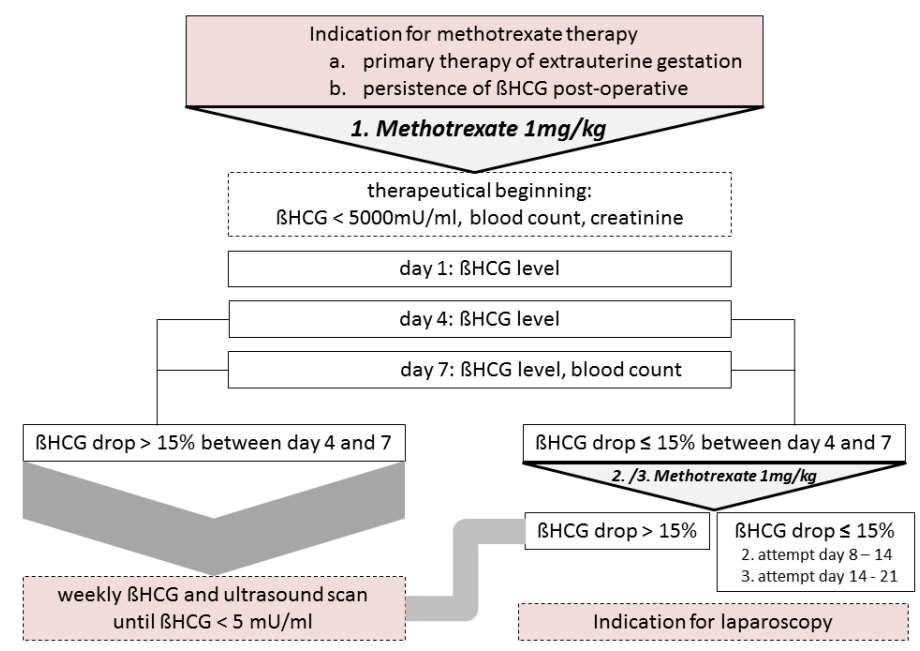

Figure 3. Algorithm of Methotrexate Therapy. 
Although this type of operation is gentle and organ saving, it has a higher rate of incomplete removal and, therefore, a higher risk of recurrence and of trophoblast residuals.

\section{Salpingostomy}

The majority of ectopic pregnancies located in the infundibulum have to be operated either by salpingectomy or by segmental tubectomy and secondary reanastomosis after a certain interval. However, approx. $8 \%$ of women have persistent ectopic pregnancy after salpingostomy. This has to be kept in mind and be part of the preoperational discussion (2).

Before opening the tube, vasopressin, 20 IU diluted in 100 $\mathrm{mL}$ of normal saline, is injected into the mesosalpinx over the mesenteric surface of the tubal segment containing the gestational products. As the trophoblast has a high cell division, its metabolic rate is equivalently high.

Evacuation by Aquadissection or Aspiration, Luxation and Preparation

A 1-2 cm longitudinal incision is made on the most distended part of the antimesenteric tubal wall, which is often of bluish discoloration. Usually it is possible to identify the different tubal wall layers: serosa, muscularis externa and interna and the mucosa. Once the lumen is open, the friable gestational sac bulges out of the wound and can be evacuated by aspiration. If the product of conception is surrounded by blood clots, it must be extracted through the tubal incision with alternating pressurized suction and irrigation or with the aid of a grasping or biopsy forceps. The site of implantation is then irrigated extensively. The irrigation fluid must drain from both sites, the salpingotomy and the fimbrial end. Minimal bleeding from the tubal bed is normal and ceases in most cases spontaneously.

The decision to suture after salpingotomy is taken by the surgeon. Usually the incision does not require suturing. Suturing can be recommended if bleeding occurs and requires extensive coagulation. If the defect has to be closed, a continuous suture or single knots approximating the edges with single 4-0 absorbable sutures are made. Nevertheless, the follow-up and prognosis for recurrent extra uterine pregnancies is not improved after suturing.

\section{Partial Salpingectomy}

If salpingostomy cannot resolve the problem, a partial salpingectomy can be tried before salpingectomy is performed. Indications for a partial salpingectomy are tubal rupture, a pregnancy located in the isthmus or a recurrent tubal pregnancy. In most cases of isthmic pregnancy a linear salpingotomy is unsuccessful as these gestations grow through the lumen of the tube and into the tunica muscularis; therefore, segmental resection is recommended.

Coagulation of the tubal part is done by bipolar forceps on both ends of the affected part including the corresponding mesosalpinx. After resecting the affected part of the tube the mesosalpinx is cauterized stepwise. The partial salpingectomy can be completed by reanastomosis.

\section{Salpingectomy}

The indications for salpingectomy include no desire for future pregnancies, recurrent tubal pregnancies and the occurrence of an extra uterine pregnancy after a failed sterilization or a previously reconstructed tube. Other indications for salpingectomy are made intraoperatively, for example severe adhesions, hydrosalpinx, tubal rupture, persistent bleeding after a safe tubal procedure or if the tubal pregnancy is over $5 \mathrm{~cm}$ in diameter.

It is of utmost importance to resect the tube within the utero-tubal junction to avoid cornual pregnancies after IVF if further pregnancy is desired.

An ectopic pregnancy is defined as extraluminal when the gestational product is situated between the muscularis externa and the serosa. Incision with the monopolar needle or hook over the point of maximum distention results in the product of conception slipping out without the need of a long incision.

\section{Surgical Technique for Nontubal Ectopic Pregnancy Ovarian Pregnancy}

The unusual site and rarity of ovarian pregnancies leads to a more complex clinical course. The difficulty in making an early and accurate diagnosis can lead to an inconsistent therapeutic approach with an unpredictable outcome and a life-threatening status if the ovary ruptures.

(a) Superficial pattern: The implantation takes place in an ovarian focus of endometriosis.

(b) Intrafollicular pattern (90\% of ovarian pregnancies):

- Primary - insemination of an ovum that has not yet ovulated.

- Secondary - after regular ovulation implantation of the inseminated ovum in the follicle or corpus luteum.

Tubal pregnancy, hemorrhagic ovarian cyst, endometrioma and other pelvic diseases have the same clinical findings as ovarian pregnancies. Therefore, the diagnosis is quite difficult.

The traditional operative treatment for ovarian pregnancies has been oophorectomy. However, the desire to maintain reproductive capability and improvements in laparoscopy has more recently led to the ovarianpreserving operational technique. By enucleating the gestational sac bluntly from the ovary, the surrounding ovarian tissue is protected to the greatest possible extent.

\section{Abdominal Pregnancy}

Abdominal pregnancy can be either primary or secondary in case of rupture of a tubal pregnancy and abdominal implantation. This rare localization occurs in only about $1 \%$ of all ectopic pregnancies. Nevertheless, it has a high morbidity and mortality rate and makes detection by ultrasound or magnetic resonance imaging (MRI) necessary.

Due to comparatively few symptoms the abdominal gravidity is often recognized very late. The treatment of 
an advanced abdominal pregnancy is associated with an enormous risk for life-threatening maternal bleeding. The removal of the placenta is dependent on its localization as it can be implanted on any organ in the abdominal cavity. Sometimes the placenta is better left intraabdominally to be calcified and reabsorbed or embolized by an interventional radiologist prior to removal at a second intervention. Its management is strictly by laparotomy.

Interstitial or Cornual Pregnancy/Rudimental Uterine Horn If a pregnancy implants in the interstitial area of the fallopian tube, we speak of an interstitial or cornual ectopic pregnancy (incidence 1\%-3\% of extrauterine pregnancies). The interstitial line has been described as an echogenic line extending from the cornual side of the endometrium into the cornual region, reaching the ectopic gestational sac, and is said to be a highly specific sign for interstitial pregnancy. Its mortality rate is about $2 \%$. This is due to the high vascularity of this area where the uterine and ovarian vessels join together. The classical treatment methods are laparotomy, uterine horn resection or even hysterectomy (29).

The cornual pregnancy, implants in the same anatomical area of the tube but opens to the uterine cavity. Therefore, the operative method of choice can be hysteroscopy. To avoid uterine perforation, larger pregnancies can be removed by curettage under laparoscopic guidance.

\section{Cesarean Scar Ectopic Pregnancy}

Deficient uterine scars are a frequent finding in women with a history of previous cesarean section. The risk of scar deficiency is increased in women with a retroflexed uterus and in those who have undergone multiple cesarean sections.

Early diagnosis can offer treatment options to avoid uterine rupture and hemorrhage. Available data suggest that termination of pregnancy is the treatment of choice in the first trimester soon after the diagnosis. Expectant treatment in these cases is not indicated (30). Laparoscopy enables the successful treatment of an unruptured ectopic pregnancy in a previous Cesarean scar and makes it possible to preserve the patient's reproductive capability. Figure 10 clearly demonstrates the striking diagnosis with a Doppler ultrasound.

Viable Cesarean scar pregnancies have been treated safely by selective transarterial embolization in combination with subsequent dilatation and curettage and local or systemic injections of MTX. Hysteroscopic management has also been reported.

\section{Intraligamental Gravidity}

For intraligamental development (1 in 250 ectopic pregnancies) the gestational sac must split the oviduct precisely between the leaves of the broad ligament. With an intact amnion, the embryo develops in its extraperitoneal sac. Rupture occurs early enough so that the villi are capable of expanding their areas of nidation.
Cervical and Vaginal Pregnancy

The criteria for a cervical pregnancy have been described by Rubin in 1911:

1. Cervical glands must be present opposite the placental attachment.

2. The attachment of the placenta to the cervix must be intimate.

3. The placenta must be below the peritoneal reflection of the anterior and posterior surfaces of the uterus.

4. Fetal elements must not be within the uterine cavity.

Cervical and vaginal pregnancies are threatening localizations for the patient. Due to their anatomic closeness to the uterine artery they connect early to the corresponding drainage. Diagnostic detection of a cervical pregnancy should implicate a local or systemic medical treatment with methotrexate. The medical treatment can be accompanied by selective uterine artery embolization. It is therefore essential to know the sonomorphology of cervical ectopic pregnancy: homogene decidual reaction, closed inner cervical os and round gestational sac.

An isthmic localisation is associated with a severe risk for uncontrollable intraoperative bleeding; however, this can be handled well by laparoscopy. Rarely is a laparotomy necessary.

\section{Simultaneous Intra- and Extrauterine Pregnancy}

Simultaneous intra- and extrauterine pregnancy has increased with the wide application of artificial reproductive technologies (ART).

In the majority of cases the simultaneous pregnancy is an incidental finding. Persistent abdominal pain or other clinical features alongside an irregular rise in the $ß-H C G$ level can lead to this infrequent diagnosis. Once a simultaneous pregnancy has been discovered, the operative method of choice is laparoscopic salpingectomy.

Bilateral tubal pregnancy is diagnosed and treated during single laparoscopy following clinical and sonographic diagnosis of tubal ectopic pregnancy of mostly only one side. Before the undetected second ectopic pregnancy becomes clinically obvious, persisting beta HCG levels after the initial surgery raise suspicion.

\section{Discussion}

Complications

Precedent bleedings are caused by the invasion of trophoblast tissue into the local vascular system leading to intraluminal hematoma and bleeding of the fimbrial end. If the stretching capacity of the tube is exceeded, this results in tubal rupture.

It should be emphasized that the possibility of leaving behind products of conception is the same after laparoscopy as after laparotomy (5\%-15\%). Attention should be focussed at surgery on the medial portion of the tube as this is the preferential site where trophoblastic tissue can survive and be responsible for elevated serum B-HCG levels.

At times the differential diagnosis of ectopic pregnancies is difficult. They can be of gynecological origin (for example 
early stage of normal pregnancy, miscarriage, intrauterine abortion, torsion of ovary or fallopian tube, ovarian cyst, ruptured corpus luteum cyst or follicle, necrotic myoma, pelvic inflammatory disease and tubo-ovarian abscess) or of non-gynecological origin (for example acute appendicitis; cystitis; pyelonephritis; nephrolithiasis; perforation of hollow organs, such as stomach, bowel or gallbladder; obstruction of hollow organs; intraabdominal inflammation; rupture of parenchymatous organs, such as liver, spleen or kidney; vascular ischemic disease and vascular hemorrhagic disease) (20).

The B-HCG levels after operative treatment should be reduced by $70 \%$ after two days and by another $70 \%$ after seven days. About $30 \%$ of women with a previous ectopic pregnancy have difficulties conceiving. The conception rate is about $77 \%$, no matter what kind of therapy they receive. The incidence of recurrence is between $5 \%$ and $20 \%$ and rises up to $32 \%$ following two ectopic pregnancies $(18,31)$.

Today, it appears useful to perform routine early ultrasound scans of all women with positive pregnancy tests to check the location of the gestation so that earlier and more conservative treatment options are possible in cases of ectopic pregnancies. Nevertheless, the presence of an intrauterine pregnancy does not exclude the possibility of a heterotopic pregnancy as pregnancies at multiple locations may occur (Figure 4).

\section{Ethical Issues}

Not applicable.

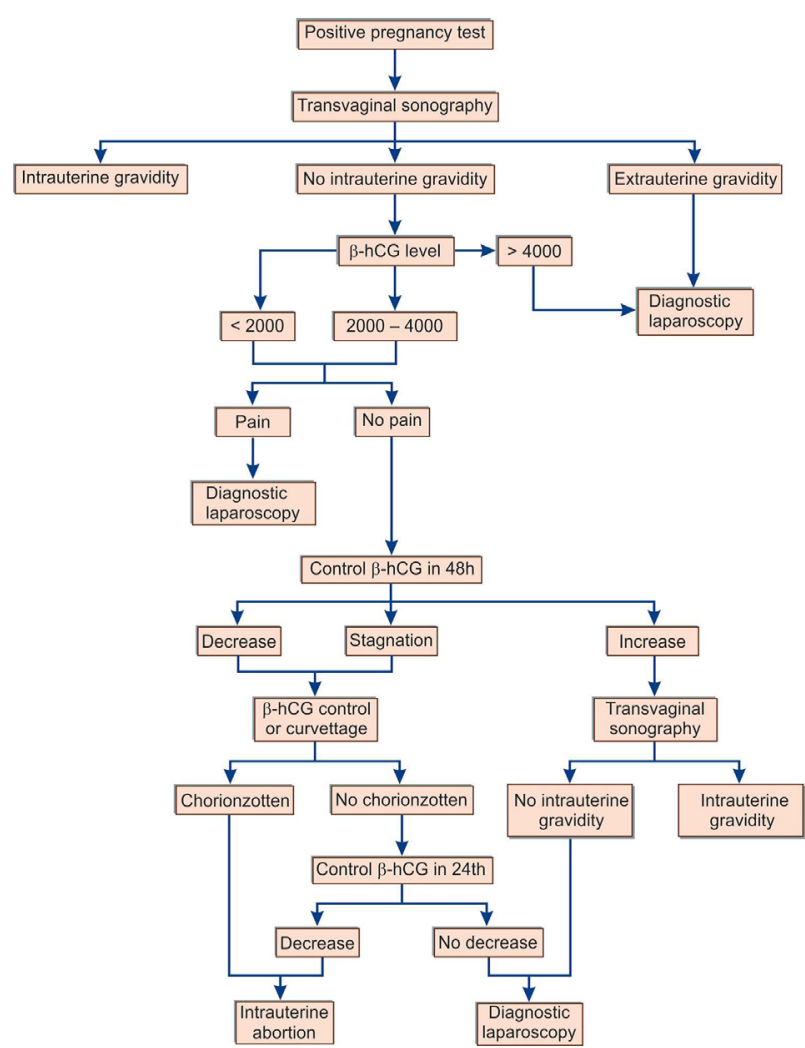

Figure 4. Flowchart on Diagnostic and Operative Procedure in Case of Potential Ectopic Pregnancy.

\section{Conflict of Interests}

The authors declare that they have no competing interests.

\section{Financial Support}

The researchers received no financial support or grant from any funding agency in the public and commercial sectors.

\section{Acknowledgments}

The authors thank Dawn Rüther for editing the manuscript.

\section{References}

1. Bouyer J, Coste J, Fernandez H, Pouly JL, Job-Spira N. Sites of ectopic pregnancy:a 10 year population-based study of 1800 cases. Hum Reprod. 2002;17:3224-3230. doi:10.1093/ humrep/17.12.3224.

2. Murray H, Baakdah H, Bardell T, Tulandi T. Diagnosis and treatment of ectopic pregnancy. CMAJ. 2005;173:905-912. doi:10.1503/cmaj.050222.

3. Dericks-Tan JS, Scholz C, Taubert HD. Spontaneous recovery of ectopic pregnancy:a preliminary report. Eur J Obstet Gynecol Reprod Biol. 1987;25:181-185. doi:10.1016/0028-2243(87)90097-9.

4. Lipscomb GH, Stovall TG, Ling FW. Nonsurgical treatment of ectopic pregnancy. N Engl J Med. 2000;343:1325-1329. doi:10.1056/NEJM200011023431807.

5. Cheng X, Tian X, Yan Z, Jia M, Deng J, Wang Y, et al. Comparison of the fertility outcome of salpingotomy and salpingectomy in women with tubal pregnancy:a systematic review and meta-analysis. PloS One. 2016;11:e0152343. doi:10.1371/journal.pone.0152343.

6. Refaat B, Dalton E, Ledger WL. Ectopic pregnancy secondary to in vitro fertilisation-embryo transfer: pathogenic mechanisms and management strategies. Reprod Biol Endocrinol. 2015;13:30. doi:10.1186/s12958015-0025-0.

7. $\mathrm{Bu} \mathrm{Z,} \mathrm{Xiong} \mathrm{Y,} \mathrm{Wang} \mathrm{K,} \mathrm{Sun} \mathrm{Y.} \mathrm{Risk} \mathrm{factors} \mathrm{for} \mathrm{ectopic}$ pregnancy in assisted reproductive technology: a 6-year single-center study. Fertil Steril. 2016;106:90-94. doi:10.1016/j.fertnstert.2016.02.035.

8. Kadar N, Romero R. Serial human chorionic gonadotropin measurements in ectopic pregnancy. Am J Obstet Gynecol. 1988;158:1239-1240. doi:10.1016/0002-9378(88)90263-3.

9. Fritz MA, Guo SM. Doubling time of human chorionic gonadotropin (hCG) in early normal pregnancy: relationship to hCG concentration and gestational age. Fertil Steril. 1987;47:584-589. doi:10.1016/S00150282(16)59107-9.

10. Brennan DF. Ectopic pregnancy--Part I: clinical and laboratory diagnosis. Acad Emerg Med 1995;2:1081-1089. doi:10.1111/j.1553-2712.1995.tb03154.x.

11. Kurjak A, Zalud I, Schulman H. Ectopic pregnancy: transvaginal color Doppler of trophoblastic flow in questionable adnexa. J Ultrasound Med. 1991;10:685-689.

12. Honemeyer U. Primary care in obstetrics and gynecology - A place for advanced ultrasound. Donald School Journal of Ultrasound in Obstetrics and Gynecology. 2009;3:61-74. doi:10.5005/jp-journals-10009-1022.

13. Carson SA, Buster JE. Ectopic pregnancy. N Engl J Med. 1993;329:1174-1181. doi:10.1056/NEJM199310143291608.

14. Ramakrishnan K, Scheid DC. Ectopic pregnancy:forget the 
"classic presentation" if you want to catch it sooner. J Fam Pract. 2006;55:388-395.

15. Sivalingam VN, Duncan WC, Kirk E, Shephard LA, Horne AW. Diagnosis and management of ectopic pregnancy. J Fam Plann Reprod Health Care. 2011;37:231-40. doi:10.1136/ jfprhc-2011-0073.

16. Ardaens Y, Guerin B, Perrot N, Legoeff F. Contribution of ultrasonography in the diagnosis of ectopic pregnancy. J Gynecol Obstet Biol Reprod (Paris). 2003;32:S28-S38.

17. Mehta TS, Levine D, Beckwith B. Treatment of ectopic pregnancy:is a human chorionic gonadotropin level of 2,000 $\mathrm{mIU} / \mathrm{mL}$ a reasonable threshold? Radiology. 1997;205:569573. doi:10.1148/radiology.205.2.9356647.

18. Lozeau AM, Potter B. Diagnosis and management of ectopic pregnancy. Am Fam Physician. 2005;72:1707-1714.

19. Mol BW, Lijmer JG, Ankum WM, van der Veen F, Bossuyt PM. The accuracy of single serum progesterone measurement in the diagnosis of ectopic pregnancy:a metaanalysis. Hum Reprod. 1998;13:3220-3227. doi:10.1093/ humrep/13.11.3220.

20. Alkatout I, Honemeyer U, Strauss A, et al. Clinical Diagnosis and treatment of ectopic pregnancy. Obstet Gynecol Surv. 2013;68(8):571-581. doi:10.1097/OGX.0b013e31829cdbeb

21. Berry J, Davey M, Hon MS, Behrens R. A 5-year experience of the changing management of ectopic pregnancy. J Obstet Gynaecol. 2016;36:631-634. doi:10.3109/01443615.2015.11 33578.

22. Koike H, Chuganji Y, Watanabe H, Kaneko M, Noda S, Mori N. Conservative treatment of ovarian pregnancy by local prostaglandin F2 alpha injection. Am J Obstet Gynecol. 1990;163:696. doi:10.1016/0002-9378(90)91260-J.

23. Lang PF, Weiss PA, Mayer HO, Haas JG, Honigl W.
Conservative treatment of ectopic pregnancy with local injection of hyperosmolar glucose solution or prostaglandin-F2 alpha: a prospective randomised study. Lancet. 1990;336:78-81. doi:10.1016/01406736(90)91593-Y.

24. Shamma FN, Schwartz LB. Primary ovarian pregnancy successfully treated with methotrexate. Am J Obstet Gynecol. 1992;167:1307-1308. doi:10.1016/S00029378(11)91706-2.

25. Chelmow D, Gates E, Penzias AS. Laparoscopic diagnosis and methotrexate treatment of an ovarian pregnancy:a case report. Fertil Steril. 1994;62:879-881. doi:10.1016/S00150282(16)57020-4.

26. Alkatout I. Ectopic pregnancy. In: Schollmeyer T, Mettler L, Rüther D, Alkatout, I, eds. Practical Manual for Laproscopic \& Hysteroscopic Gynecological Surgery. 2nd ed. New Delhi: Jaypee Brothers Medical Publishers (P) Ltd; 2013:169-189.

27. Alkatout I, Stuhlmann-Laeisz C, Mettler L, Jonat W, Schollmeyer T. Organ-preserving management of ovarian pregnancies by laparoscopic approach. Fertil Steril. 2011;95(8):2467-2470.e2. doi:10.1016/j. fertnstert.2010.12.060.

28. Hoover KW, Tao G, Kent CK. Trends in the diagnosis and treatment of ectopic pregnancy in the United States. Obstet Gynecol. 2010;115:495-502. doi:10.1097/ AOG.0b013e3181d0c328.

29. Advincula AP, Senapati S. Interstitial pregnancy. Fertil Steril. 2004;82:1660-1661. doi:10.1016/j.fertnstert.2004.08.015.

30. Ash A, Smith A, Maxwell D. Caesarean scar pregnancy. BJOG 2007;114:253-263. doi:10.1111/j.1471-0528.2006.01237.x.

31. Tay JI, Moore J, Walker JJ. Ectopic pregnancy. BMJ. 2000;320:916-919. doi:10.1136/bmj.320.7239.916.

Copyright ( 2017 The Author (s); This is an open-access article distributed under the terms of the Creative Commons Attribution License (http://creativecommons.org/licenses/by/4.0), which permits unrestricted use, distribution, and reproduction in any medium, provided the original work is properly cited. 